\title{
Achievable Magnetic Fields of Super-Ferric Helical Undulators for the ILC
}

\author{
S.H. Kim \\ Advanced Photon Source, Argonne National Laboratory
}

Abstract - The magnetic fields on the beam axis of helical undulators for the proposed International Linear Collider (ILC) gamma-ray production were calculated for undulator periods of $10 \mathrm{~mm}$ and $12 \mathrm{~mm}$. The calculation assumed the use of low-carbon steel for the magnetic poles and a beam chamber outer diameter of $6.3 \mathrm{~mm}$. Using $\mathrm{NbTi}$ superconducting coils at $4.2 \mathrm{~K}$, the on-axis field for a 10 -mm-period undulator was $0.62 \mathrm{~T}$ at the critical current density. The field for a 12-mm undulator period was $0.95 \mathrm{~T}$, which gives a $K$ value of 1.06 . The $K$ value for an $11-\mathrm{mm}$ undulator with $N b_{3} S n$ superconducting coils was estimated to about 1.1 .

A transverse periodic helical magnetic field may be generated on the axis of a doublehelix coil with equal currents in opposite directions in each helix [1]. An ideal helical field may be expressed as

$$
\mathbf{B}(z)=\left\{\hat{x} B_{0} \sin (k z)+\hat{y} B_{0} \cos (k z)\right\}
$$

where $B_{0}$ is the magnetic field modulus on the undulator axis, and $k=2 \pi / \lambda$ with $\lambda$ as the undulator magnetic period length along the electron-beam direction on the $z$-axis. The radiated photon energy $e_{n}$ for the $n$th harmonic is given by

$$
e_{n}(k e V)=n \frac{9.498 E^{2}(G e V)}{\lambda(m m)\left\{1+K^{2}+(\gamma \theta)^{2}\right\}}
$$

with $E$ as the electron beam energy, $\gamma$ is the relativistic factor of the electron energy, and $\theta$ is the angle between the z-axis and the radiated photon beam direction. The deflection parameter $K$ is defined as

$$
K=0.0934 \lambda(\mathrm{mm}) B_{0}(T)
$$

From Eq. (2), the photon energy may be calculated. Currently, the proposed International Linear Collider (ILC) lists $\lambda=10 \mathrm{~mm}$ and $K=1$ for the helical undulator parameters [2]. For $K=1$, the spectral power density of the photon beam is maximum at $\gamma \theta$ $=1$ for a single electron [1]. This note calculates the fields on the undulator axis with a beam chamber outer diameter of $6.3 \mathrm{~mm}$ for periods of $10 \mathrm{~mm}$ and $12 \mathrm{~mm}$ to evaluate whether the listed $K$ value could be achieved.

Figure 1 shows a double-helix model coil for the low-carbon steel poles and the beam chamber. A double-helix superconducting (SC) coil with equal currents in opposite directions in each helix was inserted in between the steel coils for the field calculations. A 
typical calculation for the on-axis fields corresponding to Eq. (1) is plotted in Fig. 2. The calculation used OPERA-3d [3]; the $B(H)$ data of "low-carbon" steel included in the software were used for the steel poles shown in Fig. 1.

In Fig. 3, on-axis field modulus $B_{0}$ (right axis) calculated for periods $\lambda=10 \mathrm{~mm}$ and 12 $\mathrm{mm}$, and the corresponding maximum fields in the coil (left axis) are plotted as a function of the average current density in the coil. The average critical current densities $J_{c}(\mathrm{NbTi})$ and $J_{c}\left(\mathrm{Nb}_{3} \mathrm{Sn}\right)$ at $4.2 \mathrm{~K}$, for the $\mathrm{NbTi}$ and $\mathrm{Nb}_{3} \mathrm{Sn}$ SC coils (bottom axis), respectively, are functions of the coil maximum field (left axis) and limit the coil current densities and the on-axis fields. The figure shows that, at $J_{c}(\mathrm{NbTi})$ around $1.15 \mathrm{kA} / \mathrm{mm}^{2}$, the on-axis fields are approximately $0.55 \mathrm{~T}(K=0.51)$ and $0.95 \mathrm{~T}(K=1.06)$ for $\lambda=10 \mathrm{~mm}$ and $12 \mathrm{~mm}$, respectively. The $J_{c}\left(\mathrm{Nb}_{3} \mathrm{Sn}\right)$ for $\lambda=10 \mathrm{~mm}$ is about $1.9 \mathrm{kA} / \mathrm{mm}^{2}$, which gives an on-axis field of $0.85 \mathrm{~T}(K=0.8)$. From the figure, one could estimate an achievable $K$ value of about 1.1 for $\lambda=11 \mathrm{~mm}$.

When the operating current density is close to the critical current density $J_{c}(\mathrm{NbTi})$ (see Fig. 3), the stability margins of the device may become an issue and may be simulated with some form of heat loads from inside the beam chamber [4]. For the development of planartype SC undulators at the Advanced Photon Source (APS), an average $J_{c}(\mathrm{NbTi})$ up to 1.4 $\mathrm{kA} / \mathrm{mm}^{2}$ was achieved for a 14.5-mm-period short section. Considering the coil geometry for the helical undulator, $J_{c}(\mathrm{NbTi})$ and $J_{c}\left(\mathrm{Nb}_{3} \mathrm{Sn}\right)$ in Fig. 3 were reduced by $20 \%$ from that used in ref. [4]. The stability margin at the operating current density for $\lambda=12 \mathrm{~mm}$ may be enhanced by using an $\mathrm{Nb}_{3} \mathrm{Sn}$ coil.

This work was supported by the U.S. Department of Energy, Office of Science, Office of Basic Energy Sciences, under Contract No. W-31-109-ENG-38.

\section{References}

[1] B.M. Kincaid, J. Appl. Phys. 48, 2684 (1977).

[2] http://www.linearcollider.org/cms/

[3] OPERA-3d, Vector Fields Ltd., Oxford, England. The author does not imply that similar software by other vendors cannot perform the work.

[4] S.H. Kim et al., IEEE Trans. Appl. Supercond. 15, 1240 (2005); S.H. Kim et al., R\&D of ShortPeriod NbTi and $\mathrm{Nb}_{3} \mathrm{Sn}$ Superconducting Undulators for the APS, Proc. 2005 PAC, 2410 (2005). 


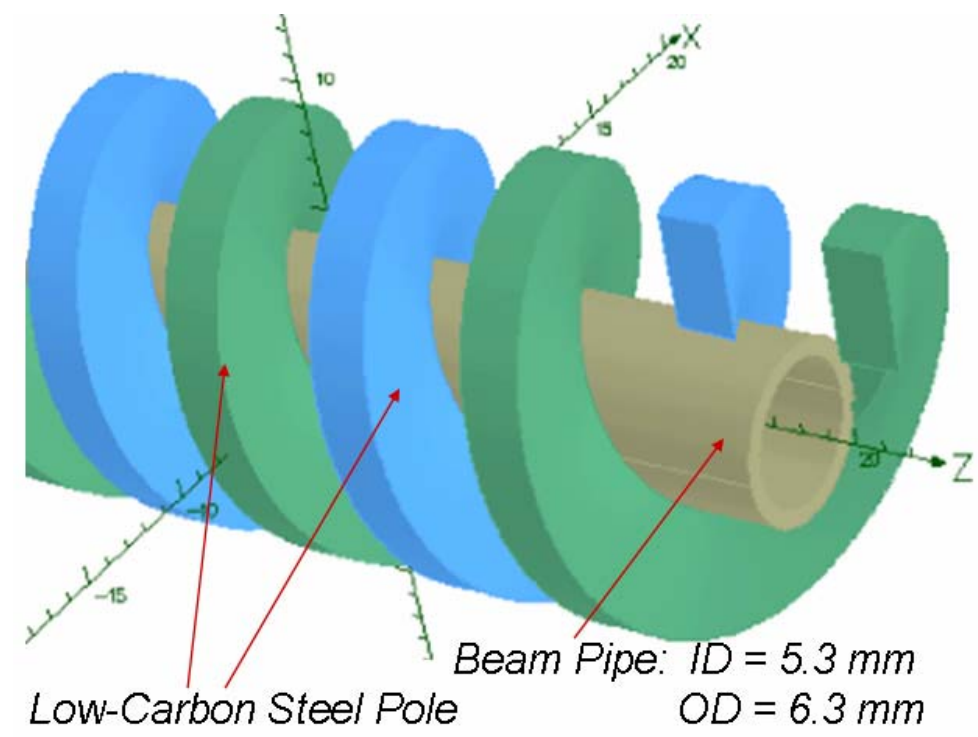

Fig. 1. A model of a double-helix coil for the low-carbon steel poles and beam chamber. A double-helix SC coil with equal currents in opposite directions in each helix is to be inserted between the steel coils.

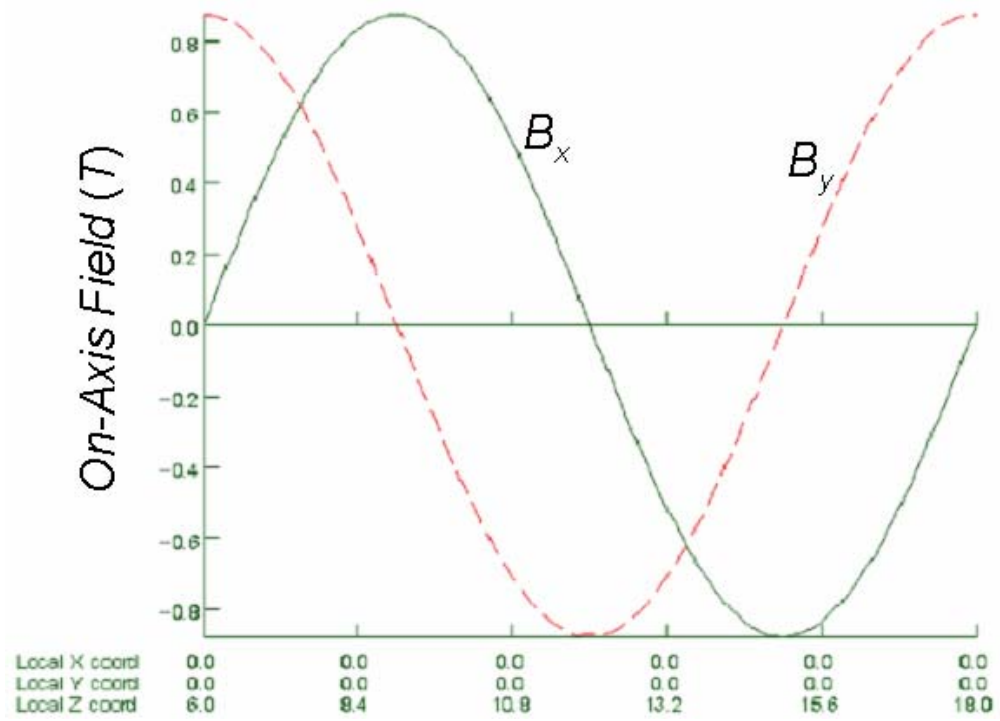

Fig. 2. Plots of calculated $B_{x}$ and $B_{y}$, two components in Eq. (1), for one 12-mm period along the undulator axis. The on-axis field $B_{0}$ was $0.88 \mathrm{~T}$ for an average coil current density of $1 \mathrm{kA} / \mathrm{mm}^{2}$ and the beam chamber outer diameter as in Fig. 1. 


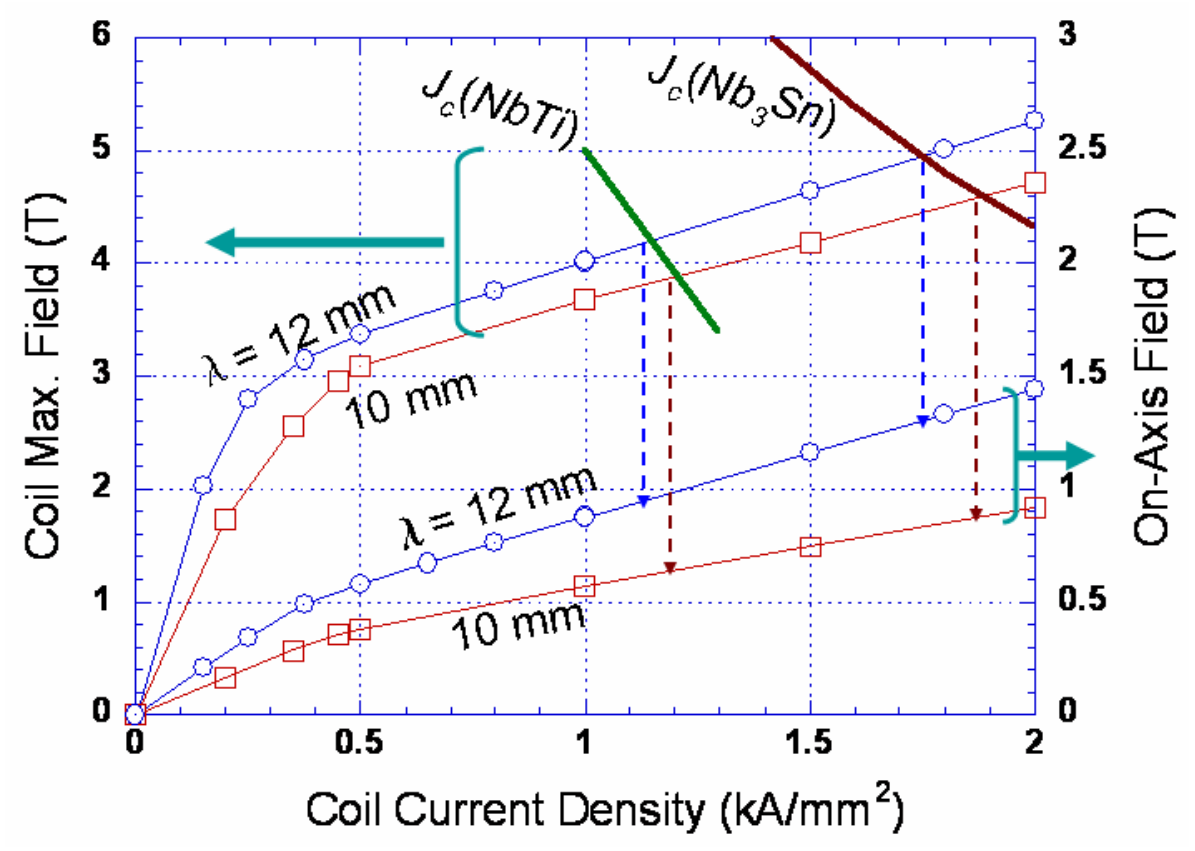

Fig. 3. On-axis field modulus $B_{0}$ (right axis) calculated for periods $\lambda=10 \mathrm{~mm}$ and $12 \mathrm{~mm}$, and the corresponding maximum fields in the coil (left axis) are plotted as a function of the average current density in the coil. The average critical current densities $J_{c}(\mathrm{NbTi})$ and $J_{c}\left(\mathrm{Nb}_{3} \mathrm{Sn}\right)$ at $4.2 \mathrm{~K}$, for the $\mathrm{NbTi}$ and $\mathrm{Nb}_{3} \mathrm{Sn}$ SC coils (bottom axis), respectively, are functions of the coil maximum field (left axis) and limit the coil current densities and the on-axis fields. 